\title{
Black hole remnants
}

\author{
Ali H. Chamseddine, ${ }^{a, b}$ Viatcheslav Mukhanov ${ }^{b, c, d}$ and Tobias B. Russ ${ }^{b}$ \\ ${ }^{a}$ Physics Department, American University of Beirut, \\ Beirut, Lebanon \\ ${ }^{b}$ Theoretical Physics, Ludwig Maxmillians University, \\ Theresienstr. 37, Munich 80333, Germany \\ ${ }^{c}$ MPI for Physics, \\ Foehringer Ring 6, Munich 80850, Germany \\ ${ }^{d}$ School of Physics, Korea Institute for Advanced Study, \\ Seoul 02455, Korea \\ E-mail: chams@aub.edu.1b, viatcheslav.Mukhanov@lmu.de, \\ Tobias.Russ@physik.uni-muenchen.de
}

ABSTRACT: We show that in asymptotically free mimetic gravity with limiting curvature the black hole singularity can be resolved and replaced by a static patch of de Sitter space. As a result of Hawking evaporation of these non-singular black holes, there remain stable remnants with vanishing Hawking temperature.

Keywords: Black Holes, Classical Theories of Gravity, Spacetime Singularities

ARXIV EPRINT: 1908.03498 


\section{Contents}

1 Introduction 1

2 The Lemaître coordinates 1

3 Modified mimetic gravity 3

4 Asymptotic freedom at limiting curvature 4

5 Exact solution $\quad 4$

$\begin{array}{llr}6 & \text { Black hole thermodynamics } & 6\end{array}$

$\begin{array}{lll}7 & \text { Conclusions } & 7\end{array}$

\section{Introduction}

The two unresolved issues of black hole physics actively discussed during the last 50 years are: a) the singularity problem, b) the final state of black hole evaporation and, closely related to it, the information paradox (see, for instance, [1]). Both issues are usually referred to some, as yet unknown, non-perturbative theory of quantum gravity. The nature of Planck mass black holes, their stability and the resolution of the space-like singularity remain unclear. In this letter we suggest a completely different approach to these problems. Namely, we assume that classical General Relativity is modified at curvatures close to, but still below Planckian curvature, in such a way that some limiting curvature, which is a free parameter of the theory, can never be exceeded (cf. [2, 3]). Moreover, we propose that at this limiting curvature the gravitational constant vanishes. These two assumptions allow us to avoid all problems related to non-perturbative quantum gravity effects and study in a fully controllable way the final stage of non-singular evaporating black holes. To implement the above ideas, we use the mimetic field introduced in [4] and further exploited in references [5-10]. We show that in asymptotically free theories with limiting curvature, black holes generically have stable remnants with mass determined by the inverse limiting curvature value, thus exceeding Planck mass. These remnants have vanishing Hawking temperature and, by the arguments shown in [10], metric quantum fluctuations never become relevant for them.

\section{The Lemaître coordinates}

The metric of both black hole and de Sitter universe in "static" coordinates can be written as

$$
\mathrm{d} s^{2}=\left(1-a^{2}(r)\right) \mathrm{d} t^{2}-\frac{\mathrm{d} r^{2}}{\left(1-a^{2}(r)\right)}-r^{2} \mathrm{~d} \Omega^{2},
$$


where $\mathrm{d} \Omega^{2}=\mathrm{d} \vartheta^{2}+\sin ^{2} \vartheta \mathrm{d} \varphi^{2}$. For a black hole of mass $M$ the function $a^{2}(r)=r_{g} / r$, where $r_{g}=2 M$ and for the de Sitter universe $a^{2}=(H r)^{2}$ with $H^{-1}$ being the radius of curvature. Throughout this paper we use Planck units where all fundamental constants are set to unity. In both cases the static coordinate system is incomplete. Moreover, at the horizon corresponding to $a^{2}=1$, there is a coordinate singularity. Therefore, in both cases it is more convenient to use the synchronous Lemaître coordinates

$$
T=t+\int \frac{a}{1-a^{2}} \mathrm{~d} r, \quad R=t+\int \frac{\mathrm{d} r}{a\left(1-a^{2}\right)},
$$

which are non-singular on the horizons (cf. [11]). In these coordinates metric (2.1) becomes

$$
\mathrm{d} s^{2}=\mathrm{d} T^{2}-a^{2}(x) \mathrm{d} R^{2}-b^{2}(x) \mathrm{d} \Omega^{2},
$$

where $a^{2}$ and $b^{2}=r^{2}$ must be expressed in terms of Lemaître coordinates $T$ and $R$ using the relation

$$
x \equiv R-T=\int \frac{\mathrm{d} r}{a(r)},
$$

which follows from (2.2). Note that the norm of the Killing vector field $\partial / \partial t=\partial / \partial R+\partial / \partial T$ vanishes wherever $a=1$. The black hole metric in these new coordinates becomes

$$
\mathrm{d} s^{2}=\mathrm{d} T^{2}-\left(x / x_{+}\right)^{-2 / 3} \mathrm{~d} R^{2}-\left(x / x_{+}\right)^{4 / 3} r_{g}^{2} \mathrm{~d} \Omega^{2},
$$

and it is regular at the horizon $x=x_{+}=4 M / 3$. The region $x>0$ covers both interior and exterior of the black hole and $x=0$ corresponds to the physical space-like singularity where curvature invariants blow up. Hence, in General Relativity this metric is not extendable to negative $x$.

Correspondingly, the de Sitter metric takes the form

$$
\mathrm{d} s^{2}=\mathrm{d} T^{2}-\exp \left(2 H\left(x-x_{-}\right)\right)\left(\mathrm{d} R^{2}+H^{-2} \mathrm{~d} \Omega^{2}\right),
$$

where $x_{-}$is a constant of integration in (2.4) and the de Sitter horizon occurs at $x=x_{-}$. The region $x<x_{-}$corresponds to the patch of size $r=H^{-1}$ covered by static coordinates, which on larger scales do not exist.

Calculating the spatial curvature of constant $T$ hypersurfaces of metric (2.3), one finds that it vanishes if $a^{2}=(\mathrm{d} b / \mathrm{d} x)^{2}$. Hence, both solutions (2.5) and (2.6) are spatially flat in the Lemaitre slicing.

The Schwarzschild metric has a Kasner type space-like singularity. In the previous paper [10] we have found that in a theory where the ideas of limiting curvature and asymptotic freedom of gravity are realized via the mimetic field, Kasner singularities are avoided and replaced by a de Sitter region at limiting curvature. In this paper we implement these same ideas for black holes. Using ansatz (2.3) for the metric, we will find an explicit solution describing a non-singular black hole whose metric approaches asymptotics (2.5) and (2.6) at low and high curvatures, respectively. 


\section{$3 \quad$ Modified mimetic gravity}

Introducing the mimetic field $\phi$ through a Lagrange multiplier constraint, consider the theory of gravity defined by the action

$$
S=\frac{1}{16 \pi} \int \mathrm{d}^{4} x \sqrt{-g}\left(-\mathcal{L}+\lambda\left(g^{\mu \nu} \phi_{, \mu} \phi_{, \nu}-1\right)\right),
$$

with Lagrangian density

$$
\mathcal{L}=f(\square \phi) R+(f(\square \phi)-1) \widetilde{R}+2 \Lambda(\square \phi),
$$

where

$$
\widetilde{R} \equiv 2 \phi^{, \mu} \phi^{, \nu} G_{\mu \nu}-(\square \phi)^{2}+\phi^{; \mu \nu} \phi_{; \mu \nu}
$$

and $G_{\mu \nu}$ is the Einstein tensor. In [10] we argued that $\square \phi$ is the unique measure of curvature that $f$ can depend on without introducing higher time derivatives to the modified Einstein equation. The extension of the action presented in [10] by the $\widetilde{R}$-term is done with the purpose to remove higher spatial and mixed derivatives. Thus, (3.1) defines a theory of gravity free of any higher derivatives.

Since the Lemaitre coordinates are synchronous, the generic solution of the constraint equation $\phi^{, \alpha} \phi_{, \alpha}=1$ satisfied by the mimetic field, which is compatible with the symmetries of ansatz (2.3), is $\phi=T+$ const. In this coordinate system

$$
\square \phi=\kappa=\frac{\partial}{\partial T} \ln \sqrt{\gamma}=-\frac{\mathrm{d}}{\mathrm{d} x} \ln \left(a b^{2}\right)
$$

represents the trace of extrinsic curvature $\kappa=\kappa_{a}^{a}$ of synchronous slices of constant $T$. The expression $\widetilde{R}$, given in (3.3), is nothing but the spatial curvature scalar ${ }^{3} R$ of these slices, expressed in covariant form.

Variation of (3.1) with respect to the metric $g_{\mu \nu}$ yields the modified vacuum Einstein equations. Solving the equation obtained by varying (3.1) with respect to $\phi$ for the Lagrange multiplier $\lambda$ and substituting the metric ansatz (2.3), after lengthy but straightforward calculations we find that the spatial components of the modified Einstein equations have the first integral (see [10]), ${ }^{1}$

$$
\frac{\dot{b}}{b}-\frac{\dot{a}}{a}=\frac{3 M}{f a b^{2}}
$$

where dot denotes the derivative with respect to $x$ and a constant of integration has been fixed to match the Schwarzschild solution with mass $M$ in the limit $x \rightarrow \infty$. In deriving (3.5) we have assumed that the spatial curvature remains negligible everywhere for solutions matching the two spatially flat asymptotics (2.5) and (2.6). Later on we will justify this assumption.

Accordingly, the temporal modified Einstein equation becomes

$$
\frac{\kappa^{2}\left(f-2 \kappa f^{\prime}\right)-3\left(\Lambda-\kappa \Lambda^{\prime}\right)}{\left(f+\kappa f^{\prime}\right)}=\left(\frac{3 M}{f a b^{2}}\right)^{2},
$$

where prime denotes the derivative with respect to $\kappa$. The equations (3.5) and (3.6) determine the two unknown functions $a(x)$ and $b(x)$.

\footnotetext{
${ }^{1}$ The more general and detailed calculations will be presented in a forthcoming publication by the authors.
} 


\section{Asymptotic freedom at limiting curvature}

The inverse running gravitational constant is represented by

$$
f(\square \phi)=\frac{1}{G(\square \phi)},
$$

normalized as $f(\square \phi=0)=1$ in Planck units. Asymptotic freedom is characterized by a divergence of $f$ as $\square \phi \rightarrow\left|\kappa_{0}\right|$ approaches the limiting curvature $\kappa_{0}$, which is a free parameter of the theory. In [10] we have shown that in a contracting Kasner universe the vanishing gravitational constant very efficiently suppresses anisotropies and the solution close to the limiting curvature becomes isotropic, approaching a de Sitter universe with $H=\kappa_{0} / 3$. Since black hole and Kasner singularities are similar, one can expect that the black hole singularity can be resolved in the same way. Namely, the asymptotic solution far away from the black hole, which is still given by (2.5), will start to approach (2.6) as soon as the curvature approaches its limiting value. The full solution extends for the entire range $-\infty<x<+\infty$.

For the Schwarzschild solution (2.5) the function $a \propto b^{-1 / 2}$ increases as $b \rightarrow 0$, while for the de Sitter solution $a \propto b$. It follows that $\dot{a}$ has to vanish at some intermediate point $x_{*}$ where $a$ reaches its maximum value before starting to decrease as we go deeper into the black hole. If $a\left(x_{*}\right)>1$, there exist two Killing horizons where $a\left(x_{ \pm}\right)=1$ at $x_{+}>x_{*}$ and $x_{-}<x_{*}$, named in analogy with (2.5) and (2.6). In the limiting case where $a\left(x_{*}\right)=1$, the two horizons merge at $x_{+}=x_{-}=x_{*}$ and the region where $\partial / \partial t=\partial / \partial R+\partial / \partial T$ is spacelike disappears. This is the case of a minimal black hole with mass $M_{\min } \sim \kappa_{0}^{-1}$ which exceeds the Planck mass if the limiting curvature is below the Planckian value. For $M<M_{\min }, a$ is always smaller than unity, no horizon occurs and thus no black holes with mass smaller than $M_{\min }$ exist.

One can easily find that for the metric (2.3) the surface gravity of the Killing horizons is given by

$$
g_{s}=-\dot{a}\left(x_{ \pm}\right),
$$

and it hence vanishes for the minimal black hole. Because the Hawking radiation temperature is proportional to $g_{s}$, it is equal to zero for these minimal mass black holes and as a result of evaporation there must remain stable remnants of mass $M_{\min }$. Thus, the existence of limiting curvature combined with asymptotic freedom of gravity generically leads to the existence of minimal stable black hole remnants.

To demonstrate this in a concrete theory, below we will find an explicit spatially flat, exact solution describing a non-singular black hole with stable remnant. ${ }^{1}$

\section{Exact solution}

Let us take

$$
f(\tilde{\kappa})=\frac{1+3 \tilde{\kappa}^{2}}{\left(1+\tilde{\kappa}^{2}\right)\left(1-\tilde{\kappa}^{2}\right)^{2}},
$$


where $\tilde{\kappa} \equiv \kappa / \kappa_{0}$ and chose $\Lambda(\kappa)$ in such a way that the square root of the branch $\kappa<0$ of (3.6) becomes

$$
\frac{-\tilde{\kappa}}{1-\tilde{\kappa}^{4}}=\frac{3 M / \kappa_{0}}{a b^{2}} .
$$

Taking the $x$ derivative of the logarithm of this equation and using (3.4), we obtain a first order differential equation for $\tilde{\kappa}(x)$ with the implicit solution

$$
-\kappa_{0} x=\frac{1}{\tilde{\kappa}}+2\left(\arctan \tilde{\kappa}-\tanh ^{-1} \tilde{\kappa}\right) .
$$

This provides a one-to-one relation between $x \in(-\infty, \infty)$ and $\tilde{\kappa} \in(-1,0)$ and hence $\tilde{\kappa}$ can be used to parametrize the solution for $a$ and $b$. After some algebra, we find that the exact solution of equations (3.5) and (5.2) parametrized in terms of $\tilde{\kappa}$ is given by

$$
\begin{aligned}
& a^{3}(\tilde{\kappa})=\frac{4 M \kappa_{0}}{3}|\tilde{\kappa}|\left(1-\tilde{\kappa}^{4}\right)\left(\frac{1+\tilde{\kappa}^{2}}{1+3 \tilde{\kappa}^{2}}\right)^{2}, \\
& b^{3}(\tilde{\kappa})=\frac{9 M}{2 \kappa_{0}^{2} \tilde{\kappa}^{2}}\left(1-\tilde{\kappa}^{2}\right)\left(1+3 \tilde{\kappa}^{2}\right) .
\end{aligned}
$$

Using (5.3) to express $\kappa$ in terms of $x$, we can easily verify that in the far exterior limit $x \rightarrow \infty, \tilde{\kappa}^{2} \rightarrow 0$ and the above solution tends to (2.5), describing a black hole of mass $M$. On the other hand, deep inside the black hole at $x \rightarrow-\infty, \tilde{\kappa}^{2} \rightarrow 1$ and we obtain asymptotic (2.6) corresponding to the de Sitter space with $H=\kappa_{0} / 3$. Thus, the obtained exact solution smoothly matches the desired asymptotics, in agreement with our general consideration above. The function $a$ reaches its maximum at $\tilde{\kappa}_{*}=-1 / \sqrt{5}$. The horizons, which occur at $a=1$, exist only if $a\left(\tilde{\kappa}_{*}\right) \geq 1$. This condition is satisfied only if

$$
M \geq M_{\min }=\frac{5^{5 / 2}}{18 \kappa_{0}}
$$

Otherwise, no horizon exists and the solution (5.3), (5.4), (5.5) describes solitonic-like objects whose metric is completely static and approaches the de Sitter metric in the center. For black holes with the minimum mass $M_{\min }$, there is only one horizon with vanishing surface gravity and hence these minimal black holes represent the stable remnants of evaporating black holes.

One can check that solution (5.3), (5.4), (5.5) satisfies $a^{2}=\dot{b}^{2}$ and hence the hypersurfaces $T=$ const. are exactly spatially flat, in complete agreement with the assumption under which it was derived. To better understand the properties of this solution, it is more illuminating to go back to the familiar singular static coordinates (2.1). From (2.4) it follows that $d r=a d x$ and therefore $a^{2}=\dot{b}^{2}$ implies that $b=r$ everywhere. Setting $b=r$ in equation (5.5t), we obtain an algebraic equation for $\tilde{\kappa}(r)$, which can be solved perturbatively and the obtained result can be substituted in (5.4) to determine $a^{2}(r)$ in the "static coordinates", where the metric is given by (2.1).

For $r \rightarrow \infty$ where $\tilde{\kappa}^{2} \ll 1$ we find the expansion

$$
1-a^{2}=1-\frac{2 M}{r}\left[1-\mathcal{O}\left(\left(\frac{r_{*}}{r}\right)^{3}\right)\right],
$$


where $r_{*}=\left(144 M / 5 \kappa_{0}^{2}\right)^{1 / 3}$ is the "radial" coordinate at which $\tilde{\kappa}_{*}=-1 / \sqrt{5}$ and the curvature becomes comparable to the limiting curvature. For large black holes with $M \gg$ $M_{\text {min }}$ the outer horizon, defined by $a\left(r_{+}\right)=1$, is located at

$$
r_{+}=2 M\left[1-\mathcal{O}\left(\left(\frac{M_{\min }}{M}\right)^{2}\right)\right],
$$

and the deviations from the Schwarzschild solution become significant only deeply "inside" the black hole at $r \ll r_{+}$. Close to the limiting curvature $\left(\tilde{\kappa}^{2} \rightarrow 1\right)$, that is for $r \ll r_{*}$, the metric is well approximated by

$$
1-a^{2}=1-(H r)^{2}\left[1-\mathcal{O}\left(\left(\frac{r}{r_{*}}\right)^{3}\right)\right]
$$

where $H=\kappa_{0} / 3$ and the inner de Sitter horizon occurs at

$$
r_{-}=H^{-1}\left[1+\mathcal{O}\left(\frac{M_{\min }}{M}\right)\right] .
$$

If the mass $M$ is comparable to the minimal mass $M_{\text {min }}$, these two asymptotics fail to describe the region close to the horizons. In the minimal case $M=M_{\min }$ inner and outer horizon coincide. Expanding $a(\tilde{\kappa})$ around its maximum at $\tilde{\kappa}_{*}$ and using (5.5) to express $\tilde{\kappa}$ in terms of $r$, we find that the near horizon metric of such a minimal black hole is given by

$$
1-a^{2} \approx \frac{10}{7}\left(1-\frac{r}{r_{*}}\right)^{2}
$$

where $r_{+}=r_{-}=r_{*}=2 \sqrt{5} / \kappa_{0}$. Note the similarity to the near horizon metric of an extremal charged Reissner-Nordström black hole.

\section{Black hole thermodynamics}

The Hawking temperature $T_{H}$ is determined by the surface gravity (4.2) at the exterior horizon $x_{+}$. For solution (5.1) we find

$$
T_{H}=\frac{g_{s}}{2 \pi}=\frac{\kappa_{0}}{6 \pi}\left|\tilde{\kappa}_{+}\right| \frac{1-5 \tilde{\kappa}_{+}^{2}}{1+3 \tilde{\kappa}_{+}^{2}},
$$

where $\tilde{\kappa}_{+}=\tilde{\kappa}\left(x_{+}\right) \in(-1 / \sqrt{5}, 0)$. Since $a\left(\tilde{\kappa}_{+}\right)=1$, we can use (5.4) to express $M$ also through $\tilde{\kappa}_{+}$as

$$
M=\frac{3}{4 \kappa_{0}\left|\tilde{\kappa}_{+}\right|\left(1-\tilde{\kappa}_{+}^{4}\right)}\left(\frac{1+3 \tilde{\kappa}_{+}^{2}}{1+\tilde{\kappa}_{+}^{2}}\right)^{2} .
$$

The formulae (6.1) and (6.2) implicitly define the relation $T_{H}(M)$. In particular, at large mass we reproduce in leading order the familiar Hawking formula

$$
T_{H}=\frac{1}{8 \pi M}\left[1+\mathcal{O}\left(\left(\frac{M_{\min }}{M}\right)^{2}\right)\right] .
$$


Instead of diverging as $M \rightarrow 0$, the temperature reaches its maximum value $T_{\max } \sim 10^{-2} \kappa_{0}$ at $\left|\tilde{\kappa}_{+}\right| \approx 0.23$ which corresponds to $M=M_{c} \approx 1.32 M_{\min }$. At this point the negative heat capacity diverges and becomes positive for $M<M_{c}$. Close to the minimal mass the temperature decreases as

$$
T_{H} \propto \sqrt{M-M_{\min }} .
$$

According to the Stefan-Boltzmann law, the rate of energy loss of a radiating body is determined by $\frac{\mathrm{d} M}{\mathrm{~d} t} \propto-T_{H}^{4} A$ where $A=4 \pi r_{+}^{2}$ is the horizon area. For an evaporating black hole close to minimal mass $A \sim M_{\min }^{2}$, and hence it will eventually approach $M_{\text {min }}$ according to $M(t)-M_{\min } \propto t^{-1}$. That is, the final product of black hole evaporation is a stable minimal remnant with $M=M_{\min }$ and vanishing Hawking temperature.

Finally, taking into account that the Bekenstein entropy of the black hole is equal to $S=A / 4=\pi r_{+}^{2}=\pi b^{2}\left(\tilde{\kappa}_{+}\right)$it is straightforward to verify that the modified first law of thermodynamics becomes

$$
G\left(\tilde{\kappa}_{+}\right) \mathrm{d} M=T_{H} \mathrm{~d} S,
$$

where $G\left(\tilde{\kappa}_{+}\right)=f^{-1}\left(\tilde{\kappa}_{+}\right)$is the value of the gravitational constant at the outer horizon.

\section{Conclusions}

We have shown that a modification of classical Einstein theory at very high curvatures, implementing asymptotic freedom at limiting curvature, can spare us from having to deal with non-perturbative quantum gravity (at least in application to cosmological and black hole problems). The existence of a sub-Planckian limiting curvature at which the gravitational constant vanishes can resolve the black hole singularity and replace it with a patch of de Sitter space (similar to [12]). Moreover, in this theory the final result of black hole evaporation are remnants whose near horizon geometry is similar to the extremal ReissnerNordström geometry. Therefore, the Hawking temperature of these remnants is equal to zero. In distinction from extremal Reissner-Nordström black holes, they do not exhibit a singularity and, because they have no charge, they are stable. As becomes clear from the maximal extension of the solution obtained above, ${ }^{1}$ these remnants can store an unlimited amount of information. This information lies in the absolute future of external observers and remains forever inaccessible for them. Hence, their degeneracy should not lead to any paradoxes in calculating physical processes observed by external observers. This suggests one of the possible ways for a resolution of the information paradox (see [1]). Moreover, the stable remnants can serve as well as Dark Matter candidates.

\section{Acknowledgments}

The work of A.H.C. is supported in part by the National Science Foundation Grant No. Phys-1518371. The work of V.M. and T.B.R. is supported by the Deutsche Forschungsgemeinschaft (DFG, German Research Foundation) under Germany's Excellence Strategy - EXC-2111 - 390814868 . 
Open Access. This article is distributed under the terms of the Creative Commons Attribution License (CC-BY 4.0), which permits any use, distribution and reproduction in any medium, provided the original author(s) and source are credited.

\section{References}

[1] W.G. Unruh and R.M. Wald, Information Loss, Rept. Prog. Phys. 80 (2017) 092002 [arXiv: 1703.02140] [INSPIRE].

[2] M.A. Markov, Limiting density of matter as a universal law of nature, JETP Lett. 36 (1982) 266.

[3] M.A. Markov and V.F. Mukhanov, De Sitter like initial state of the universe as a result of asymptotic disappearance of gravitational interactions of matter, Nuovo Cim. B 86 (1985) 97 [INSPIRE].

[4] A.H. Chamseddine and V. Mukhanov, Mimetic Dark Matter, JHEP 11 (2013) 135 [arXiv: 1308.5410] [INSPIRE].

[5] A.H. Chamseddine, V. Mukhanov and A. Vikman, Cosmology with Mimetic Matter, JCAP 06 (2014) 017 [arXiv: 1403.3961] [INSPIRE].

[6] A.H. Chamseddine and V. Mukhanov, Resolving Cosmological Singularities, JCAP 03 (2017) 009 [arXiv: 1612.05860] [INSPIRE].

[7] R. Cerulli et al., DAMA annual modulation and mirror Dark Matter, Eur. Phys. J. C 77 (2017) 83 [arXiv: 1701.08590] [INSPIRE].

[8] A.H. Chamseddine and V. Mukhanov, Ghost Free Mimetic Massive Gravity, JHEP 06 (2018) 060 [arXiv: 1805.06283] [INSPIRE].

[9] A.H. Chamseddine and V. Mukhanov, Mimetic Massive Gravity: Beyond Linear Approximation, JHEP 06 (2018) 062 [arXiv: 1805.06598] [INSPIRE].

[10] A.H. Chamseddine, V. Mukhanov and T.B. Russ, Asymptotically Free Mimetic Gravity, Eur. Phys. J. C 79 (2019) 558 [arXiv: 1905.01343] [inSPIRE].

[11] G. Lemaître, The Expanding Universe, Mon. Not. Roy. Astron. Soc. 91 (1931) 490 [InSPIRE].

[12] V.P. Frolov, M.A. Markov and V.F. Mukhanov, Through a black hole into a new universe?, Phys. Lett. B 216 (1989) 272 [INSPIRE]. 